\title{
Erratum to: On Controllability Problems of High-Order Dynamical Multi-Agent Systems
}

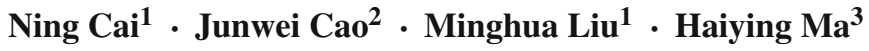

Published online: 6 May 2015

(c) King Fahd University of Petroleum \& Minerals 2015

\section{Erratum to: Arab J Sci Eng (2014) 39:4261-4267 \\ DOI 10.1007/s13369-014-0973-2}

The original version of this article unfortunately contains a mistake.

The correct form of the second paragraph in Sect. 1 should be:

"The results in the current paper form an extension to our previous works [15-18]. The novel features here are twofold: (1) A methodology is introduced for controllability analysis, which can transform a graph topology into equivalent forms. Based on this methodology, it will be shown in detail that any controllable graph with a single leader can be transformed into a path. (2) The controllability problem of heterogeneous multi-agent systems is also discussed."

The online version of the original article can be found under doi:10.1007/s13369-014-0973-2.

Ning Cai

caining91@tsinghua.org.cn

1 School of Electrical Engineering, Northwest University for Nationalities, Lanzhou 730030, China

2 Research Institute of Information Technology, Tsinghua University, Beijing 100084, China

3 School of Economics, Northwest University for Nationalities, Lanzhou 730030, China 